der Elektroden, Porosität) oder verschiedener Materialien gemacht werden (siehe Abbildung 2). Die mathematische Modellierung und Simulation ermöglicht dadurch qualitative und quantitative Vorhersagen zum Verhalten von elektrokatalytischen Zellen und trägt damit wesentlich zum Verständnis und zur systematischen Weiterentwicklung bei.
Literatur

[1] J. Newman and K. Thomas. Electrochemical Systems. John Wiley \& Sons, 2014

[2] M. Landstorfer, J. Electrochem. Soc., 2017, 164, 3671-3685

[3] S. de Groot and P. Mazur. Non-Equilibrium Thermodynamics. Dover Publications, 1984

[4] U. Hornung. Homogenization and Porous Media. Springer Science \& Business Media, 2012 der $\mathrm{MATH}^{+}$Application Area 2 - Materials, Light, Devices.

\title{
Mathematik in der Flugplanung
}

\author{
Kamal Al-Bawani, Marco Blanco Sandoval, Ralf Borndörfer, Anton Kaier, \\ Pedro Maristany, Adam Schienle und Swen Schlobach
}

\begin{abstract}
Der Verkehr ist ein Gebiet, in dem die Rolle der Mathematik auf vielfältige Weise sichtbar wird. Autos sind heute mit Routenplanungssystemen ausgestattet, demnächst werden sie autonom fahren. Kein Bahnkunde schaut heute noch im Kursbuch nach einer Verbindung. Internethändler betreiben vollautomatische Hochregallager und schaffen es, Bestellungen am gleichen Tag auszuliefern. In all diesen Systemen wird Mathematik immer mehr nicht nur zur Optimierung genutzt, sie wird selbst zum wesentlichen Bestandteil des Systems, ohne den nichts mehr geht. Das gilt auch für die Planung von Flugrouten.
\end{abstract}

Flugrouten richten sich stark nach dem Wetter: Die Maschinen starten oder landen nicht nur in die Richtung, aus der der Wind weht, auch die restliche Flugroute wird angepasst, um im Jetstream mitzugleiten, um Turbulenzen auszuweichen oder der Aschewolke des Eyjafjallajökull. Auf diese Weise werden Sicherheit und Komfort erhöht, hauptsächlich aber Treibstoff gespart, pro Route bis zu 2,5 Prozent, das ist bei den niedrigen Margen im Luftverkehr sehr viel.

Daher ist die Berechnung des Treibstoffverbrauchs die Basis jeder Routenoptimierung. Prinzipiell verbraucht ein Flugzeug weniger Kerosin, je höher es fliegt, je mehr Rückenwind es hat und je leichter es ist. Insbesondere verringert sich der Verbrauch beim Durchfliegen eines Streckenabschnittes oder "Segmentes“ kontinuierlich. Zur Berechnung geben die Flugzeughersteller für jeden Typ mit einer „specific range" genannten Funktion $f: \mathbb{R}_{+} \rightarrow \mathbb{R}+$ die marginale Reichweite in Abhängigkeit vom aktuellen Gesamtgewicht an (die Höhe und die Temperatur spielen ebenfalls eine Rolle), vgl. Abbildung 1. Deren Stammfunktion

$$
F\left(w_{\text {curr }}\right)=\int_{w_{\text {empty }}}^{w_{\text {curr }}} f(v) \mathrm{dv}
$$

gibt dann die Reichweite an, die das Flugzeug bei einem momentanen Gewicht $w_{\text {curr }}$ hat, bis der Tank beim Gewicht $w_{\text {empty }}$ leer ist. Das Endgewicht $w_{\text {end }}$ eines Flugzeugs beim Durchfliegen eines Segmentes der Länge $d$, und damit der Treibstoffverbrauch, ergibt sich aus der Gleichung

$$
\begin{aligned}
d & =\int_{w_{\text {end }}}^{w_{\text {curr }}} f(v) \mathrm{dv} \\
& =F\left(w_{\text {curr }}\right)-F\left(w_{\text {end }}\right) \Leftrightarrow w_{\text {end }} \\
& =F^{-1}\left(F\left(w_{\text {curr }}\right)-d\right),
\end{aligned}
$$

die man performant und mit kontrolliertem Fehler über eine stückweise Linearisierung der Funktion $F$ löst.

Das Wetter geht in diese Rechnung durch eine Verlängerung oder Verkürzung der Segmentlänge $d$ ein, die das Flugzeug im umgebenden Luftraum über Grund zurücklegt. Da man nicht weiß, zu welchem Zeitpunkt man in ein Segment einfliegt und wie dann das Wetter ist, muss diese Korrektur während der Berechnung „on the fly“ gemacht werden. Dies lässt sich gut mit der zeitlichen Interpolation der Wetterprognose verbinden, die in Abständen von drei Stunden vorliegt. 

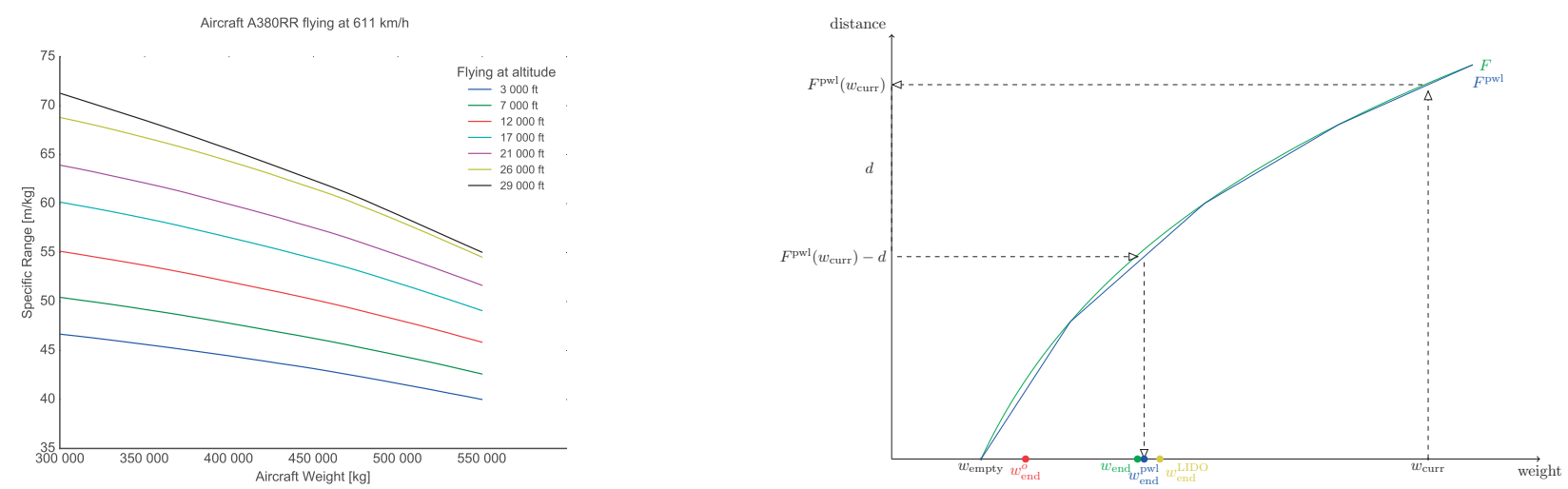

Abbildung 1. Die specific range eines Flugzeuges nimmt mit der Höhe zu und mit dem Gewicht ab (links). Der Treibstoffverbrauch kann durch stückweise Linearisierung des Integrals der specific range berechnet werden.

Im Moment findet der meiste Flugverkehr noch auf einem großen weltweiten Graphen statt, dem Airway Network. Dieser Graph hat in jedem von 40 diskreten „Flight Leveln" 60o ooo-70o ooo Kanten, die bereits bekannten Segmente (Kanten für den Steig- und Sinkflug werden zusätzlich dynamisch erzeugt), die Knoten heißen „Waypoints“. Das Flugplanungsproblem stellt sich darauf als ein zeitabhängiges Kürzeste-Wege-Problem dar.

In den letzten Jahren hat sich herausgestellt, dass die Effizienz von Beschleunigungsmethoden zur Wegesuche stark von der Problemstruktur abhängt, die sich wiederum aus dem Anwendungskontext ergibt. Im Gegensatz zum Straßenverkehr funktionieren im Luftverkehr Contraction Hierarchies und Hub Labeling nicht so gut. Vielleicht etwas überraschend kann man aber mit einem $A^{*}$-Ansatz superschnelle Laufzeiten erzielen, wenn man den richtigen Unterschätzer verwendet. Diesen bestimmt man mit Hilfe von „super-optimalem Wind“. Das ist ein artifizieller Wind, der auf einem Segment(stück) in einem gegebenen Zeitintervall den maximal auftretenden prognostizierten Rückenwind überschätzt, vgl. Abbildung 2. In der Basisversion lassen sich damit Flugrouten im Millisekundenbereich bestimmen, ausreichend, um mit professionellen Systemen wie $\mathrm{LIDO}_{4} \mathrm{D}$ von Lufthansa Systems in einem übergeordneten Branchand-Bound-Verfahren „Traffic Flow Restrictions“ (Verkehrs- regeln im Luftraum), Überflugkosten, Mindestabstände zu Ausweichflughäfen, „Fuel Policies“ und noch einiges mehr zu behandeln.

Aktuell verändert sich die Flugplanung durch die Einführung von „Free Flight“ Zonen, vgl. Abbildung 3. Immer mehr Länder erlauben dabei über ihrem Gebiet eine Abweichung der Flugrouten vom Airway Network. Eine Free Flight-Zone darf man zumeist auf gerader Linie (eigentlich auf einem Großkreis) zwischen Paaren von „Entry“und „Exit"-Points durchqueren und solche Segmente zu einer „Free Route“ verketten; langfristig soll ein echter „Free Flight" mit beliebigen Polygonzügen möglich sein (die Polygone sind der Funktionsweise der Autopiloten geschuldet, die beim Erreichen eines programmierten Punktes einen neuen, geradlinigen Kurs setzen).

Die Behandlung von Free Routes mit einem klassischen, diskreten Ansatz führt zu einer immensen Vergrößerung des Planungsgraphen und einer dementsprechenden Steigerung der Komplexität. Zurzeit kann diese Entwicklung noch durch den Einsatz zunehmend raffinierterer Beschleunigungstechniken kompensiert werden. „Combing“ ist eine solche Methode, die den Suchraum durch eine Berechnung komplexerer unterer Schranken stark reduziert. Langfristig erfordert Free Flight aber die Entwicklung von neuartigen diskret-kontinuierlichen Methoden.
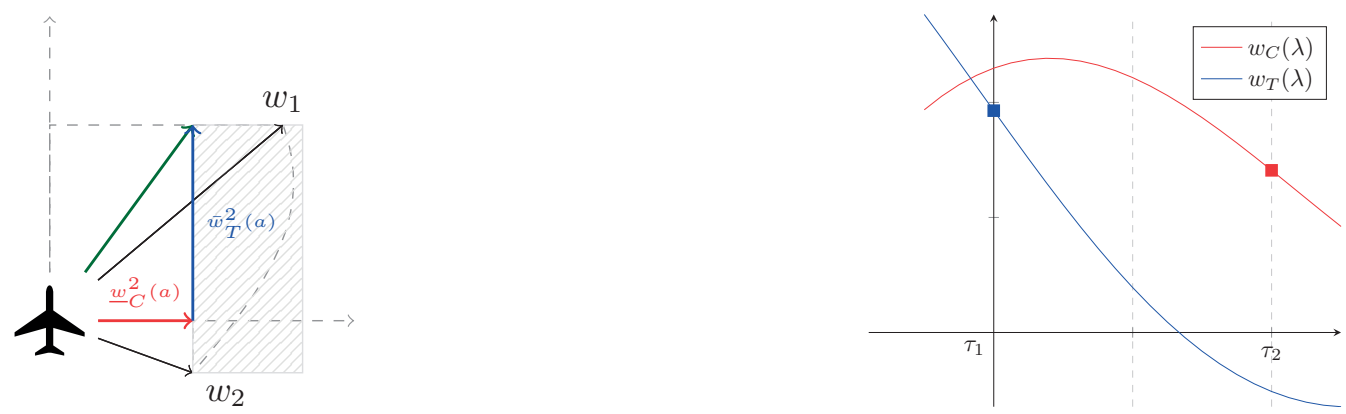

Abbildung 2. Beim Durchfliegen eines Segmentes verändert oder „dreht sich“ der Wind vom Vektor $\mathbf{w}_{1}$ zum Vektor $\mathbf{w}_{2}$, entsprechend verändern sich der Seitenwind $\mathbf{w}_{\mathbf{C}}$ („Crosswind“) und der Rückenwind $\mathbf{w}_{\mathbf{T}}$ („Trackwind“). Die Kombination des bestmöglichen Rücken- und Seitenwindes in einem Zeitintervall ergibt einen artifiziellen ,super-optimalen“ Wind $\mathbf{w}_{\mathbf{s}-\mathbf{o p t}}$. In einem Drittel aller Fälle ist dieser super-optimale Wind real, in der Regel weicht er nur minimal vom tatsächlichen Wind ab, er lässt sich auch sehr schnell berechnen. 

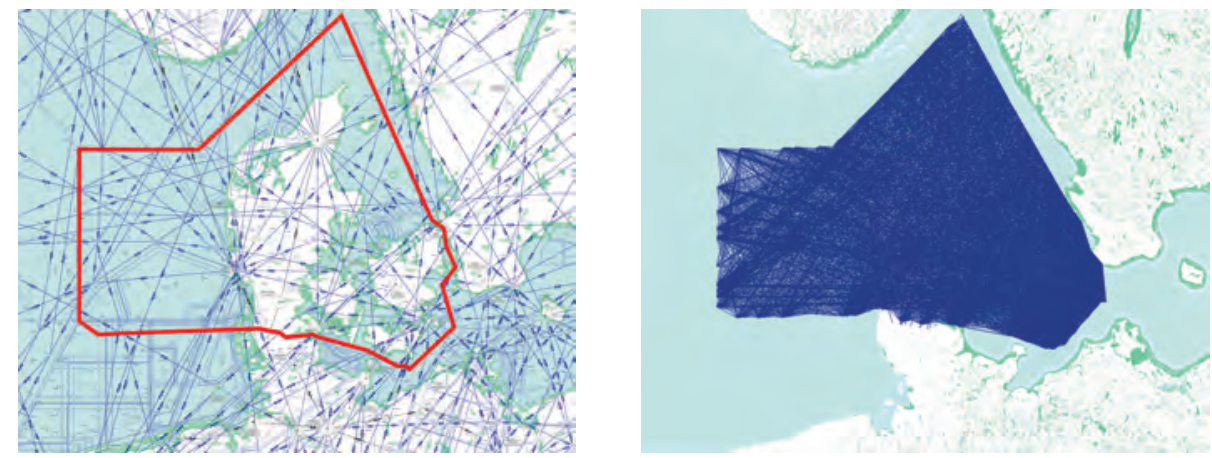

Abbildung 3. Das Airway Network über Dänemark und die neue Free Flight Zone
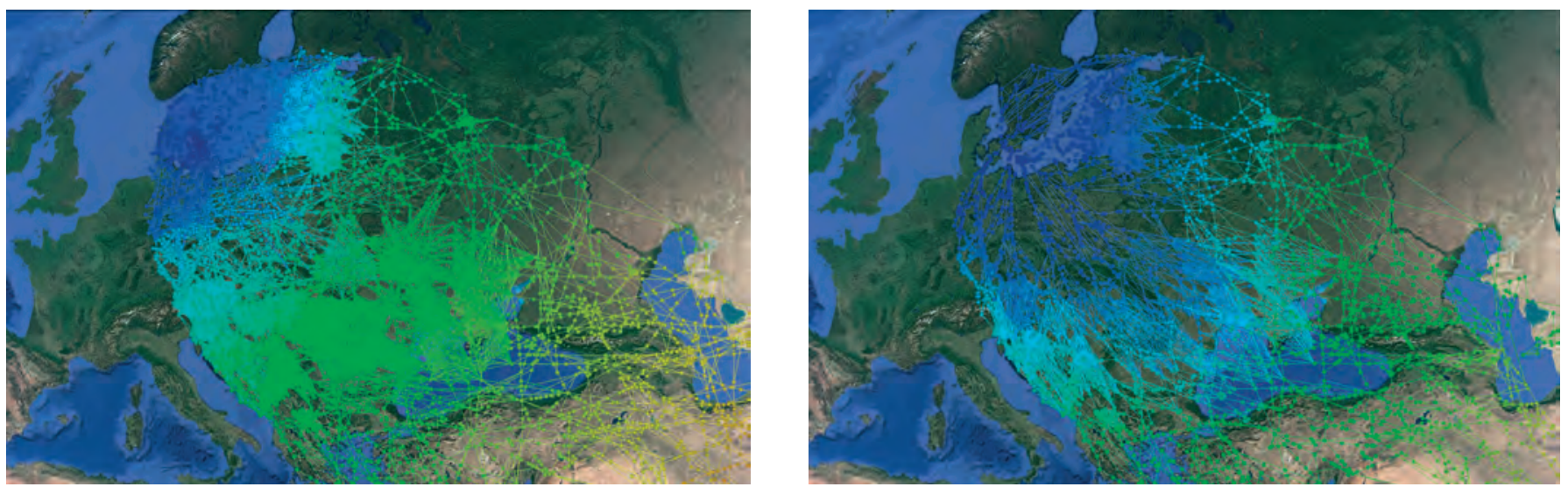

Abbildung 4. Kopenhagen-Dubai: Der Suchraum eines A*-Algorithmus bei Pruning mit super-optimalem Wind (links) und mit „combing“ (rechts). Man beachte die Ausdünnung in den Free Flight Zonen über Österreich, Ungarn und den Balkanstaaten.

In der Optimalsteuerung ist das Basisproblem der Flugplanung als Zermelo-Navigationsproblem bekannt. Es gilt als gelöst, allerdings nur, wenn man mit einer lokalen Optimallösung zufrieden ist. Das ist für die Praxis noch nicht gut genug. Es legt aber die Entwicklung hybrider Verfahren nahe, die die schnelle lokale Konvergenz kontinuierlicher Methoden mit der globalen Optimalität diskreter Verfahren kombinieren. Im MATH ${ }^{+}$-Projekt AA3-3 „DiscreteContinuous Shortest Path Methods in Flight Planning" wird $\mathrm{ab}$ dem nächsten Jahr an diesem Thema gearbeitet. Nach einer diskreten Suche in einem groben Graphen soll mit einer adaptiven direkten Methode eine kontinuierliche Optimal- lösung in einer Umgebung der diskreten Lösung gesucht werden. Eine trapezoide Kollokation mit Schrittweitenkontrolle liefert sogar die gewünschte polygonale Lösung.

Allerdings zeichnen sich weitere Herausforderungen bereits ab. Wenn - und alles deutet darauf hin - der Flugverkehr noch weiter zunimmt, können die Flugrouten bald nicht mehr einzeln geplant werden, sondern müssen kollaborativ bestimmt werden. Einige Flugzeuge müssen dann Umwege machen, damit andere besser ans Ziel kommen. Das wirft sofort spieltheoretische Fragen auf. Spätestens dann wird es auch nicht mehr ohne massiv parallele Verfahren auf Supercomputern gehen.

Prof. Dr. Ralf Borndörfer, Pedro Maristany, Adam Schienle Zuse-Institut Berlin (ZIB), Takustraße 7, 14195 Berlin borndoerfer@zib.de,maristany@zib.de,schienle@zib.de

Kamal Al-Bawani, Marco Blanco, Anton Kaier, Swen Schlobach Lufthansa Systems GmbH \& Co KG, Am Messeplatz 1, 65479 Raunheim kamal.al-bawani@lhsystems.com,marco.blanco-sandoval@lhsystems.com, anton.kaier@lhsystems.com,schlobach@lhsystems.com 